\title{
Shock intensity and warning signal effects on several measures of operant avoidance acquisition
}

\author{
ARLO K. MYERS \\ University of California, Riverside, California 92502
}

\begin{abstract}
Acquisition of both signaled and unsignaled operant avoidance learning was studied in 64 rats as a function of shock intensity, with three different warning signals used in the signaled procedure. In both signaled and unsignaled avoidance, overall response rate was a progressively increasing function of shock intensity. This was due to both an absolute and relative increase in the frequency of responses at shorter interresponse times with increasing shock intensity. Presence of an effective warning signal in the interval immediately preceding shock increased the probability of an avoidance response in this interval, decreased overall response rate, and reduced shock frequency. A buzzer signal proved most effective, followed by tone and light. However, once a warning signal occurred, the probability of an avoidance response to the signal was virtually independent of shock intensity. Also, an index of avoidance efficiency proved to be inversely related to shock intensity.
\end{abstract}

There is now a substantial body of evidence supporting a generally inverse relationship between shock intensity and acquisition performance of rats trained in discrete-trials, signaled avoidance learning in operant conditioning chambers (Bolles \& Warren, 1965; D'Amato \& Fazzaro, 1966) and in shuttle alleys (Levine, 1966; Moyer \& Korn, 1964). However, there is relatively little evidence on free operant (Sidman) avoidance performance as a function of shock intensity. Powell (1970) reported unsignaled free-operant leverpress acquisition to be an increasing function of shock intensity and, in a somewhat related experimental operation, Stone (1966) found such performance to be an increasing function of the duration of discrete shocks employed. There is also some evidence that the maintenance of performance on overtrained avoidance tasks is an increasing function of shock intensity for both discrete-trials and free operant tasks (Boren, Sidman, \& Herrnstein, 1959; D'Amato, Fazzaro, \& Etkin, 1967; Huff, Piantanida, \& Morris, 1967).

The present study investigated acquisition performance in signaled and unsignaled free operant avoidance. The signaled operant task is particularly interesting because it combines features of both the traditional discrete-trials procedure and the free operant method. For example, one can extract a performance index analogous to the "percent avoidance" measure typically used in discrete-trials analysis, as well as the response rate and frequencyof-shocks measures typical in free operant work.

Three different warning signals (buzzer, tone, and

This work was supported in part by an Intramural Grant from the University of California, Riverside. The major results were presented at the meeting of the Western Psychological Association in Los Angeles on April 8, 1976. light) were used in the signaled avoidance conditions, principally because earlier work had shown very reliable differences between groups trained with these different signals in both discrete-trials and free operant avoidance (Myers, 1959, 1964). In addition, unsignaled free operant groups were trained with which to compare signaled performance. These groups also served as comparisons with existing studies of unsignaled operant avoidance as a function of shock intensity.

Two different stocks of rats were available in the local colony, and since reliable and theoretically interesting differences between rat strains had been obtained in some earlier studies (Myers, 1959, 1964), both stocks were employed.

\section{METHOD}

\begin{abstract}
Subjects
The subjects were 32 female albino rats, descendents of Sprague-Dawley stock, and 32 female hooded rats, descendants of Long-Evans stock, ranging from 95 to 150 days of age at the time of training. All subjects were born and raised in the local animal colony under essentially identical maintenance conditions. All were on ad-lib feeding up to the time of their single training session. No food or water was available during the training session. Equal numbers of albinos and hoods were assigned at random to the various experimental treatments.
\end{abstract}

\section{Apparatus}

The subjects were trained in an operant conditioning chamber $20 \times 20 \times 23 \mathrm{~cm}$ high with stainless steel walls and a shock-grill floor consisting of 3-mm-diam stainless steel rods spaced $12.5 \mathrm{~mm}$ center-to-center. There were two identical manipulanda, drum-shaped stainless steel wheels which the subject rotated treadmill-fashion with its forepaws. Each wheel was $9.5 \mathrm{~cm}$ in diameter and $5 \mathrm{~cm}$ wide, with its axis of rotation $8.2 \mathrm{~cm}$ above the grill floor, and protruded into the chamber a maximum of $2.8 \mathrm{~cm}$ through the front wall. The two wheels were located $6 \mathrm{~cm}$ to the right and left, respectively, of the midline of the chamber's front wall. 
Initiation of turning $(10 \mathrm{~g}$ minimum force through approximately $30^{\circ}$ of rotation) on either wheel qualified as a response, but continued rotation and stopping had no consequences. This is analogous to the usual method with leverpressing, in which leverdepression operates the response mechanism but sustained holding and release do not. Wheel turning has been used in a number of avoidance studies (e.g., Anderson \& Nakamura, 1964; Myers, 1959, 1962). Though it is often characterized by speedier occurrence of the very first responses and somewhat higher overall response rates than leverpressing, in many other respects the two manipulanda exhibit similar functional relations (Myers, 1959).

The tone warning signal was a $4,000-\mathrm{Hz} 70-\mathrm{dB}$ SPL pure tone presented via a ceiling loudspeaker. The buzzer was a $6-\mathrm{V}$ ac signaling buzzer (Edwards No. 115, size 1), located above the ceiling, which produced a 70-dB SPL sound intensity. The light signal was a $1-W$ incandescent lamp on the upper rear wall. It increased the illumination of the floor area to about $34 \mathrm{~cd} / \mathrm{m}^{2}$, from the $11 \mathrm{~cd} / \mathrm{m}^{2}$ provided under all other conditions by the ceiling-mounted houselight.

The various intensities of shock originated from an adjustable, constant voltage $60-\mathrm{Hz}$ ac source, through a 140 -kilohm series resistance and a Gerbrands grid scrambler. The wheels and chamber walls were wired to the scrambler as independent shock elements. The shock intensities specified are the RMS source intensities in volts.

Experimental events in the chamber were programmed by automatic electronic programming equipment, and the subject's responses were recorded by printing electrical counters.

\section{Procedure and Design}

Each subject was trained in a single $7-\mathrm{h}$ session on an operant avoidance (Sidman) schedule having a 64-sec response-shock (R-S) interval, and a 4-sec shock-shock (S-S) interval. Individual shocks were $250 \mathrm{msec}$ in duration. For those subjects having a warning signal (WS), the signal occurred at a response-WS interval of $56 \mathrm{sec}$, i.e., $8 \mathrm{sec}$ before the next shock was programmed to occur. Once it appeared, a WS remained on continuously until terminated by the subject's next response.

The experiment was a 4 by 4 by 2 factorial design consisting of all combinations of four shock source intensities (100, 150, 225 or $337.5 \mathrm{~V}$ ), four WS conditions (Buzzer, Tone, Light, or No WS), and the two animal stocks (albino or hooded rats). Two subjects were randomly assigned to each of the resulting 32 cells.

Each $1 / 4 \mathrm{~h}$ after the start of training, response totals were recorded in three mutually exclusive categories: (a) responses having interresponse times (IRTs) between 0 and $56 \mathrm{sec}$, (b) IRTs between 56 and $64 \mathrm{sec}$, and (c) IRTs greater than $64 \mathrm{sec}$. These correspond, respectively, to responses postponing both the WS interval and shock, responses in the WS interval which terminate the WS (if any) and postpone shock, and responses during the S-S period which terminate both the WS (if any) and the S-S period.

Some pilot animals (whose data are not reported as part of the present experiment) were run at a shock level of $75 \mathrm{~V}$, but $18 \%$ of these animals failed to maintain a consistent level of responding. The lowest intensity actually employed in the experiment $(100 \mathrm{~V})$ was thus close to the weakest shock capable of sustaining performance in $100 \%$ of the subjects trained. At $100 \mathrm{~V}$, shock current in this apparatus ranges from around $0.47 \mathrm{~mA}$ early in a session to around $0.29 \mathrm{~mA}$ in the later hours of a session, due to a general upward drift in the rat's contact resistance on the grill floor during the later hours of a session. This general level of shock is reasonably close to the minimally effective intensity reported by Bolles and Warren (1965) and D'Amato and Fazzaro (1966).

\section{RESULTS}

Mean frequency of responses with 0-56-sec IRT ranged between 90 and 110 for all experimental sub- groups during the first training hour. The mean for Buzzer groups exhibited a progressive decrease in 0-56-sec IRTs throughout training, whereas means for Light, Tone, and No WS groups showed a moderate increase. The two lowest shock groups $(100$ and $150 \mathrm{~V})$ also exhibited progressively decreasing 0-56-sec IRT frequencies over training hours, whereas the 225 and $337.5-\mathrm{V}$ groups progressively increased. The terminal performance resulting from these trends is shown in Figure 1, top panel, which displays final (7th) hour performance as a joint function of type of WS and shock intensity.

These final hour data were first analyzed as a 4 by 4 by 2 factorial analysis of variance for the effects of shock intensity, warning signals, rat stocks,

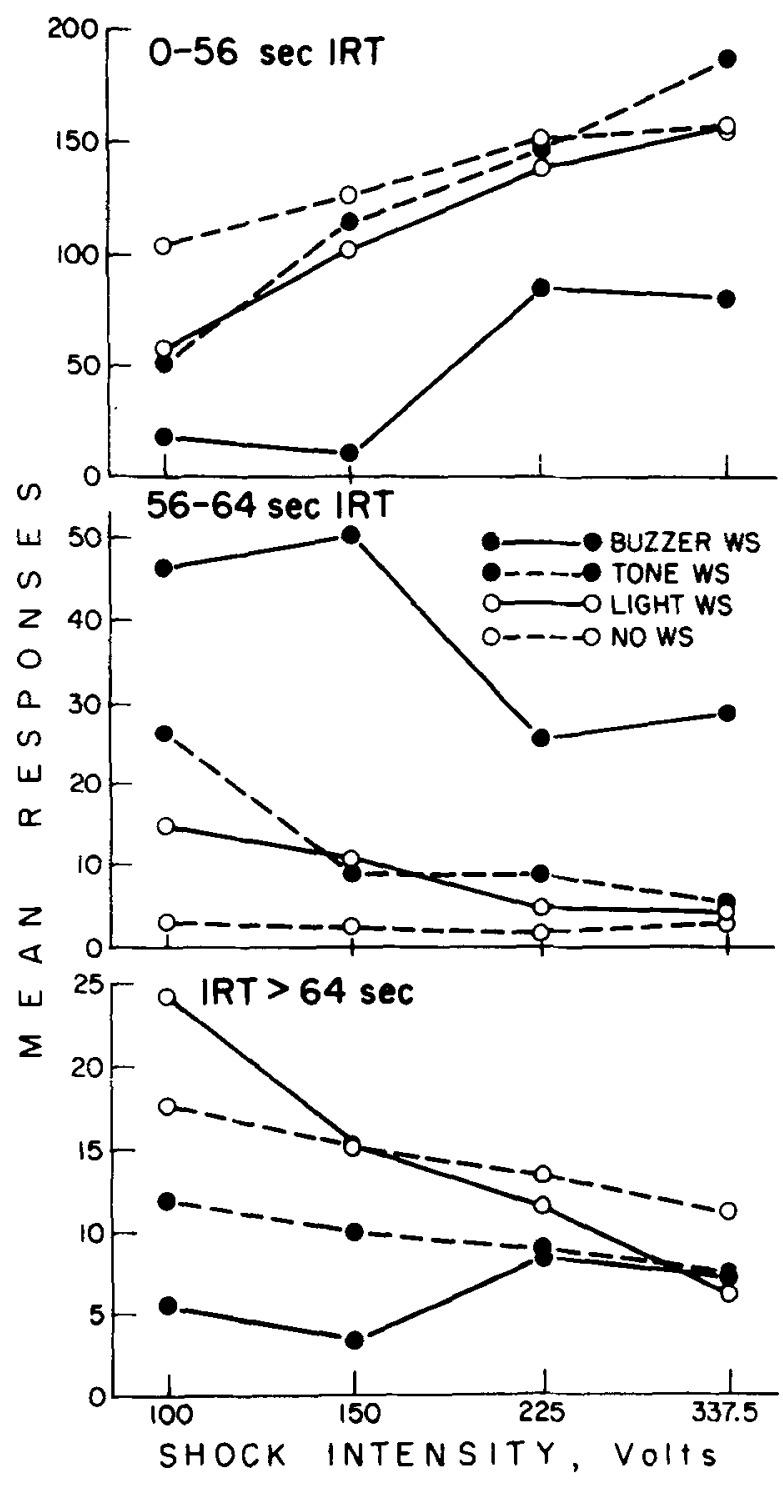

Figure 1. Mean total responses in the final training hour having 0-56-sec IRT (top), 56-64-sec IRT (middle), and IRT $>64 \mathrm{sec}$ (bottom). 
and their interactions. Performance was reliably related to both shock intensity and type of WS, but no other effect or interaction was significant. The four different shock intensities had been ordered in equal logarithmic steps of source voltage, and this factor was subjected to an orthogonal trends analysis. The linear component of the positive trend of performance vs shock intensity was highly reliable $[\mathrm{F}(1,32$; linear $)=51.90, \mathrm{p}<.001]$, with no other trend component being significant. For WS type, $\mathrm{F}(3,32)=18.19, \quad \mathrm{p}<.001$. A Newman-Keuls multiple comparisons analysis of the WS groups showed that response frequency in the Buzzer group was reliably below that in every other WS group $(\mathrm{p}<.01)$, but no other comparison was significant.

In contrast, mean responses during the WS interval (56-64-sec IRT) exhibited functional relations which were almost uniformly the opposite of those just presented for 0-56-sec IRT. Over the $7 \mathrm{~h}$ of training, the mean for the $100-\mathrm{V}$ groups exhibited substantial increases in performance on this measure, while those for the 150-, 225-, and 337.5-V groups displayed, in that order, progressively less increase over training hours. The mean of the No WS conditions showed no increase whatever throughout the training session, whereas the Light, Tone, and Buzzer conditions exhibited, in that order, progressively steeper acquisition curves.

The net result of these trends in final hour performance is shown in Figure 1, middle panel, which displays mean 56-64-sec IRTs in the 7th hour of training as a joint function of type of WS and shock intensity. Mean performance was reliably related to both shock intensity and type of WS. No other effect or interaction was statistically significant. The linear component of the negative trend of responses vs shock intensity was highly reliable $[\mathrm{F}(1,32$; linear $)=$ $19.70, \mathrm{p}<.001]$. No other trend component was statistically significant. For type of WS, $F(3,32)=$ $45.53, \mathrm{p}<.001$. The superior response-evoking potential of the buzzer, compared with tone and light (generally in that order) is consistent with earlier studies of different warning signals in avoidance conditioning (Myers, 1959, 1964.) A Newman-Keuls multiple-comparison analysis of the present WS data showed that performance in the Buzzer group was reliably greater than that in every other WS group $(p<.01)$, Tone performance was above that to No WS $(p<.05)$, but no other comparison was significant.

The mean frequency of shock periods (IRT $>64 \mathrm{sec}$ ) displayed downward trends as a function of hours of training, with the 337.5-V shock groups exhibiting the greatest trend of decrease over trials, and the $225-, 150-$, and $100-\mathrm{V}$ conditions showing, in that order, progressively less decrease. The Buzzer groups had a substantially lower mean frequency of IRTs $>64 \mathrm{sec}$ than the Tone, Light, and No WS groups from the first hour of training onward.
The final performance resulting from these trends is displayed in Figure 1, bottom panel, in which 7th $h$ performance is displayed as a joint function of shock intensity and type of WS. It can be seen that IRTs $>64 \mathrm{sec}$ were an inverse function of shock intensity and tended to be an inverse function of WS effectiveness. This measure also showed the only significant effect of rat stocks which appeared in any of the results: albino rats received fewer shock periods $(\bar{X}=8.66)$ than did hoods $(\bar{X}=13.28)$. For this comparison, $F(1,32)=8.71, p<.001$. No interactions were significant. Only the linear component of the shock intensity trend was significant $[\mathrm{F}(1,32$; linear $)=8.87, \mathrm{p}<.01]$. For WS type, $\mathrm{F}(3,32)=6.62, \mathrm{p}<.005$. In the multiple comparisons analysis, only the Buzzer-Light and Buzzer-No WS comparisons were significant $(\mathrm{p}<.01)$.

Data on total number of 250 -msec shocks also was taken for each animal over the entire 7-h session, but this measure was not recorded separately for each 15 min of training as were the other measures. Mean shock rate over all $7 \mathrm{~h}$ was reliably affected only by type of WS $[F(3,32)=7.01, p<.001]$. Mean shock rates per hour were: Buzzer, 10.78; Tone, 21.54; Light 24.12; No WS, 26.96. After the first hour of training (i.e., after subjects have acquired escape behavior from S-S periods), the measure of shocks/hour is almost identical to the measure of number of IRTs $>64 \mathrm{sec}$ (shown for the final hour in Figure 1, bottom). The reason for this is that after the earliest stages of acquisition, almost all S-S periods consist of one initial 250 -msec shock followed by a response with a latency of less than $4 \mathrm{sec}$. Therefore, an S-S period having more than one shock is rare after the first hour.

It can be seen from Figure 1 that the absolute number of responses at 0-56-sec IRT was much larger than in the other two categories. Therefore, mean total responses are highly correlated with the $0-56-\mathrm{sec}$ IRT measure. The total response measure exhibits functions very similar to those in the top panel of Figure 1, and the results of the statistical analysis of response totals is almost identical to that reported for 0-56-sec IRT. The fact that overall response rate for both signaled and unsignaled groups is an increasing function of intensity is consistent with Powell's (1970) results for unsignaled free operant training.

Presence of a WS shifts the IRT distribution so that a greater proportion of responses occur during WS intervals and fewer in pre-WS intervals. Overall response rate is reduced and the general efficiency of avoidance improves as performance becomes discriminated and controlled by the WS. This is consistent with earlier descriptions of WS effects (Myers, 1964; Sidman, 1955).

However, one significant fact about responding in the WS interval is not apparent from the data presented so far. When a subject makes a relatively 
large number of shorter latency responses, e.g., 0-56-sec IRT, it has fewer opportunities to respond at longer IRTs. Therefore, the fact that, for example, subjects trained with strong shock have fewer absolute numbers of responses in the WS interval than those trained with weak shock, may merely reflect the fewer occasions on which an IRT over $56 \mathrm{sec}$ occurs for the strong shock group, rather than a failure to perform a response once such an interval occurs. What is needed here is an index of the conditional probability of performing a response in the WS interval, provided that the opportunity occurs. This is the same index that Anger (1956) termed the "IRT/Op.," or the likelihood of response in a certain interresponse interval, given an opportunity in that interval.

For subjects having a WS, the conditional probability of responding in the WS interval is essentially the same as the traditional measure used in discretetrials signaled avoidance, i.e., the number or proportion of avoidances in successive equal blocks of training trials. The probability of response in the WS interval was computed by taking the ratio 56-64 $\mathrm{sec}$ IRTs/IRTs > $56 \mathrm{sec}$, for each subject.

This measure was generally an increasing function of hours of training, with the different shock groups showing little difference in acquisition rate or mean performance. The various WS groups, on the other hand, exhibited very substantial differences at the end of the first hour of training, differences which were only slightly augmented by the final hour of training. The mean value of this index of conditional probability of response in the WS interval for the final hour of training is displayed in Figure 2, top panel, as a joint function of shock intensity and type of WS.

It can be seen that the probability of response at 56-64-sec IRT was virtually independent of shock intensity $[F(3,32)<1.0]$. The sole factor producing a reliable effect on this measure was type of WS $[F(3,32)=33.31, p<.001]$. Multiple comparisons analysis of the WS effect showed that every WS group was reliably different from every other, the Tone-Light and Tone-No WS contrasts being significant with $\mathrm{p}<.05$, and all other contrasts being significant with $\mathrm{p}<.01$.

This failure of shock intensity to affect the probability of response to a WS seems inconsistent with the evidence cited earlier from discrete-trials training, that avoidance is better acquired at low than at high shock intensities. Furthermore, in the present signaled operant procedure, the low-shock groups have a greater number of occurrences of the WS interval than do the high-shock groups. Despite this surplus of WS "trials," they do not achieve a higher probability of response to the WS.

But suppose we analyze the performance of the present subjects in still another way. Consider the

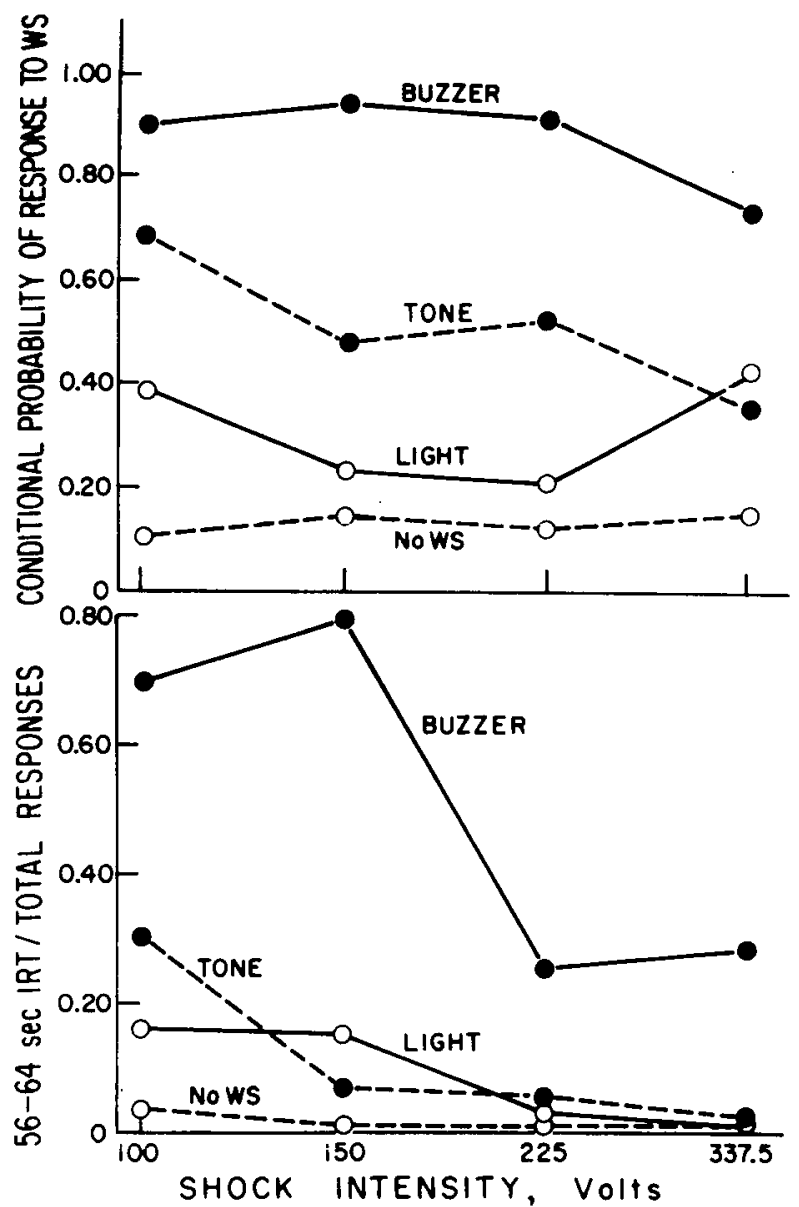

Figure 2. Top: Mean conditional probability of response in the 56-64-sec WS interval. Bottom: Mean value of the avoidance "efficiency" ratio (Responses with 56-64-sec IRT)/(Total responses). All data for the final training hour.

concept of an "ideal" asymptotic performance, in which the subject virtually always responds only in the WS interval, thus achieving maximum shock avoidance with a minimum expenditure of responses. The ratio (56-64 sec IRTs)/(total responses) would approach 1.0 in this ideal performance, and the relative magnitude of this ratio is one way of indexing the relative "efficiency" of avoidance performance.

This ratio was computed for each subject. It is generally a uniformly increasing function of hours of training, with the exception of the No WS groups, which exhibited essentially no improvement over hours of training. Performance on this index in the final hour of training is depicted in Figure 2, bottom panel, as a joint function of shock intensity and type of WS.

There were highly reliable main effects of shock intensity $[F(3,32)=13.44, p<.001]$ and type of WS $[F(3,32)=52.31, \mathrm{p}<.001]$, and a highly reliable interaction between shock intensity and WS $[F(9,32)=4.56, p<.001]$. No other effects were 
statistically reliable. Thus, in terms of this avoidance efficiency index, lower shock intensities do indeed produce better acquisition than high intensities. This trend is strongest in the Buzzer groups, weaker in the Tone and Light groups, and minimal in the No WS groups. At low shock intensities, presence of and type of WS is an important determinant of overall performance efficiency. For example, multiple comparisons among the WS groups at the 100- and $150-V$ shock levels showed that all WS groups were reliably different from each other $(p<.05$ or better $)$, with the exception of the Tone vs Light contrast. For the 225- and 337.5-V groups combined, the Buzzer condition was reliably above all others $(p<.01)$, but no other contrasts were reliable.

\section{DISCUSSION}

A reasonably clear picture of the functions of both shock intensity and type of WS in the acquisition of signaled operant avoidance emerges from the total analysis. Higher shock intensities produce a higher overall response rate, as Powell (1970) also observed. This higher rate, consisting principally of shorter IRTs, is generally sufficient to produce a moderate reduction in the frequency of exposure to shock (Figure 1, bottom). Once a WS interval occurs, however, the probability of responding in this interval is almost unaffected by shock intensity, though it is powerfully affected by the presence of a WS and the type of WS employed (Figure 2, top). The overall efficiency of acquisition performance is progressively degraded with increasing shock intensity, but is reliably improved by the presence of a WS, the benefit from a WS being greatest at the lowest shock intensities (Figure 2, bottom).

The question remains why responses with $0-56$-sec IRTs were highly positively correlated with shock intensity, while response probability in the WS interval was almost unaffected by shock intensity, and why response to a WS in other studies under similar conditions, but using discrete-trials training, is often a negative function of intensity. The following hypothesis is offered as a possible explanation.

Responding in either the typical operant or nonoperant paradigm is considered as originating via escape responses which terminate shock or reduce its density, allowing the subject to experience the avoidance contingency which then increasingly controls performance. These mechanisms are assumed to be a positive function of shock intensity. It is further assumed that although a WS does function as an instrumental discriminandum which improves avoidance efficiency, it also acquires to some degree a response-suppressive tendency as a result of being a signal for shock, this inhibitory effect also being an increasing function of shock intensity (Annau \& Kamin, 1961).
The escape/avoidance mechanisms which are positive functions of shock intensity dominate in controlling the short-IRT responding in operant schedules, so that the frequency of these (and overall response rate) is a positive function of intensity. Response to a WS in signaled operant or in discretetrials avoidance, on the other hand, is more powerfully affected by the increasingly inhibitory effects of intense shocks which cancel the effects of the first mechanism and cause performance to a WS to be little affected by, or often an inverse function of, shock intensity. This same general hypothesis could also account for the inverse relation with shock intensity found by D'Amato, Etkin, and Fazzaro (1968) for "anticipatory responses" occurring in the presence of a WS in the absence of an avoidance contingency. It is also probable that in operant avoidance, responses at longer IRTs tend to suffer the same postulated inhibitory effects as a function of increasing shock levels, accounting for the fact that even in the absence of a WS, response probability in the WS interval is little improved at higher shock intensities.

\section{REFERENCES}

Anderson, N. H., \& Nakamura, C. Y. Avoidance decrement in avoidance conditioning. Journal of Comparative and Physiological Psychology, 1964, 57, 196-204.

ANGER, D. The dependence of interresponse times upon the relative reinforcement of different interresponse times. Journal of Experimental Psychology, 1956, 52, 145-161.

Annau, Z. \& Kamin, L. J. The conditioned emotional response as a function of intensity of the US. Joumal of Comparative and Physiological Psychology, 1961, 54, 428-432.

Bolles, R. C., \& W ARREN, J. A. The acquisition of bar press avoidance as a function of shock intensity. Psychonomic Science, $1965,3,297-298$.

Boren, J. J., Sidman, M., \& Herrnstein, R. J. Avoidance, escape and extinction as functions of shock intensity. Journal of Comparative and Physiological Psychology, 1959, 52. 420-425.

D'Amato, M. R., Etxin, M., \& Fazzaro, J. Effects of shock type and intensity on anticipatory responses. Joumal of Comparative and Physiological Psychology, 1968, 66, 527-529.

D'Amato, M. R., \& Fazzaro, J. Discriminated lever-press avoidance learning as a function of type and intensity of shock. Journal of Comparative and Physiological Psychology, 1966, 61, 313-315.

D'Amato, M. R.. Fazzaro, J., \& Erkin, M. Discriminated barpress avoidance maintenance and extinction in rats as a function of shock intensity. Journal of Comparative and Physiological Psychology, 1967, 63, 351-354.

Huff, F. W., Piantanida, T. P., \& Morris, G. L. Free operant avoidance responding as a function of serially presented variations of UCS intensity. Psychonomic Science, 1967, 8, 111-112.

LEVINE, S. UCS intensity and avoidance learning. Journal of Experimental Psychology, 1966, 71, 163-164.

MoYER, K. E., \& KoRN, J. H. Effect of UCS intensity on the acquisition and extinction of an avoidance response. Journal of Experimental Psychology, 1964, 67, 352-359.

Myers. A. K. Avoidance learning as a function of several training conditions and strain differences in rats. Journal of Comparative and Physiological Psychology, 1959. 52, 381-386.

MYERS. A. K. Effects of CS intensity and quality in avoidance con- 
ditioning. Journal of Comparative and Physiological Psychology. 1962. 55. 57-61.

MyERS. A. K. Discriminated operant avoidance learning in Wistar and G-4 rats as a function of type of warning stimulus. Journal of Comparative and Physiological Psychology, 1964, 58, 453-455.

Powell. R. W. The effect of shock intensity upon responding under a multiple-avoidance schedule. Journal of the Experimental A nalysis of Behavior. 1970. 14. 321-329.

Sidman. M. Some properties of the warning stimulus in avoid- ance behavior. Joumal of Comparative and Physiological Psichologi'. 1955. 48. 444-450.

Stone. G. C. Some tactors that intluence acquisition of freeoperant avoidance behavior. Psychological Reports, 1966, 18. 383-396.

(Received for publication June 22, 1976; revision accepted September 7.1976.) 